\begin{tabular}{|c|l|}
\hline Title & Transfer of Light Helicity to Nanostructures \\
\hline Author(s) & $\begin{array}{l}\text { Toyoda, Kohei; Takahashi, Fuyuto; Takizawa, Shun; Tokizane, Yu; Miyamoto, Katsuhiko; Morita, Ryuji; Omatsu, } \\
\text { Takashige }\end{array}$ \\
\hline Citation & $\begin{array}{l}\text { Physical Review Letters, 110(14), 143603-1-143603-5 } \\
\text { https://doi.org/10.1103/PhysRevLett.110.143603 }\end{array}$ \\
\hline Issue Date & 2013-0405 \\
\hline Doc URL & http://hdl.handle.net/2115/60594 \\
\hline Rights & @2013A merican Physical Society \\
\hline Type & article \\
\hline File Information & PhysRevLett.110.pdf \\
\hline
\end{tabular}

Instructions for use 


\title{
Transfer of Light Helicity to Nanostructures
}

\author{
Kohei Toyoda, ${ }^{1}$ Fuyuto Takahashi, ${ }^{1}$ Shun Takizawa, ${ }^{1}$ Yu Tokizane,${ }^{1,3}$ Katsuhiko Miyamoto, ${ }^{1}$ \\ Ryuji Morita, ${ }^{2,3}$ and Takashige Omatsu ${ }^{1,3}$ \\ ${ }^{1}$ Graduate School of Advanced Integration Science, Chiba University, Chiba 263-8522, Japan \\ ${ }^{2}$ Department of Applied Physics, Hokkaido University, Sapporo 060-8628, Japan \\ ${ }^{3}$ Japan Science and Technology Agency, CREST, 5 Sanbancho, Chiyoda-ku, Tokyo 102-0075, Japan
}

(Received 27 November 2012; published 5 April 2013)

We discovered that chiral nanoneedles fabricated by vortex laser ablation can be used to visualize the helicity of an optical vortex. The orbital angular momentum of light determines the chirality of the nanoneedles, since it is transferred from the optical vortex to the metal. Only the spin angular momentum of the optical vortex can reinforce the helical structure of the created chiral nanoneedles. We also found that optical vortices with the same total angular momentum (defined as the sum of the orbital and spin angular momenta) are degenerate, and they generate nanoneedles with the same chirality and spiral frequency.

Light that has a helical wave front due to an azimuthal phase shift, $\exp (i L \phi)$ (where $L$ is an integer known as the topological charge), carries orbital angular momentum, $L \hbar$. Such light is referred as an optical vortex [1-4]. Optical vortices have been widely investigated for applications such as optical trapping and guiding [5-7], as well as superresolution microscopy [8,9]. Circularly polarized light has a helical electric field and a spin angular momentum, $S \hbar$, associated with its circular polarization. Optical vortices with circular polarization exhibit both wave front and polarization helicities, and a total angular momentum, $J \hbar$ [10-12], which is defined as the sum of the orbital and spin angular momenta. This angular momentum is evidenced by the orbital and spinning motions of trapped particles in optical tweezers.

To date, several researchers have intensely studied the interaction of structured light, such as radially polarized beams, with plasmonic or metallic structures [13-15]. However, these previous studies mostly focused on optical properties, such as mode selection, plasmon focusing, etc., of plasmonic or metallic structures, including photonic crystals as well as plasmonic waveguides, prepared by conventional integrated photonic circuit techniques based on lithography and chemical etching. There are few reports on the use of structured light itself to form chiral structures on the nanoscale. Recently, we discovered that the helicity of a circularly polarized optical vortex can be directly transferred to an irradiated metal sample, resulting in the formation of chiral nanoneedles [16-18]. This is the first demonstration, to the best of our knowledge, of nanostructures created by structured light with angular momenta, and it clearly represents a new scientific phenomenon.

We have also investigated control of the chirality of formed nanoneedles by changing the sign of the optical vortex helicity. Chiral nanostructures have the potential to form many new material structures [19], including planar chiral metamaterials $[20,21]$ and plasmonic nanostructures $[22,23]$. They can also be used to selectively identify the chirality of chemical composites in nanoscale imaging systems, such as atomic force and scanning tunnel microscopes [24-27].

However, it is currently unclear whether the wave front or polarization helicity (characterized by the angular momenta, $L \hbar$ and $S \hbar$, respectively) primarily contribute to chiral nanoneedle fabrication in optical vortex ablation or whether both helicities (characterized by the total angular momentum, $J \hbar$ ) have similar contributions to chiral nanoneedle formation. In this study, we investigated the structures of chiral nanoneedles fabricated using optical vortices with various values of the angular momenta $L \hbar$, $S \hbar$, and $J \hbar$ to determine which helicity of light mainly determines the chiral nanoneedle structure. We found that only the sign of the orbital angular momentum determines the chirality of the nanostructures, whereas the polarization helicity reinforces the helical structure of the formed chiral nanoneedles.

The target in the present study was a $\sim 2$-mm-thick polished tantalum $(\mathrm{Ta})$ plate, with a relatively low ablation threshold compared to other metals [28]. The pump laser was a conventional flash-lamp-pumped $Q$-switched $\mathrm{Nd}$ : YAG laser (wavelength, $1064 \mathrm{~nm}$; pulse duration, $30 \mathrm{~ns}$; pulse repetition frequency, $10 \mathrm{~Hz}$ ). A spiral phase plate (SPP) was used to shape the laser output to produce a firstorder optical vortex with $L=1$. The SPP was fabricated by electron beam etching, and it was azimuthally divided into 16 regions by using an $n \pi / 8$ phase shift (where $n$ is an integer between 0 and 15). Additionally, two overlaid SPPs were used to produce a second-order optical vortex with $L=2$. A quarter-wave plate was placed in the optical path between the SPPs and a focusing lens. It imparted spin angular momentum to the optical vortices. The sign of the optical vortex helicity was reversed by inverting the SPPs 

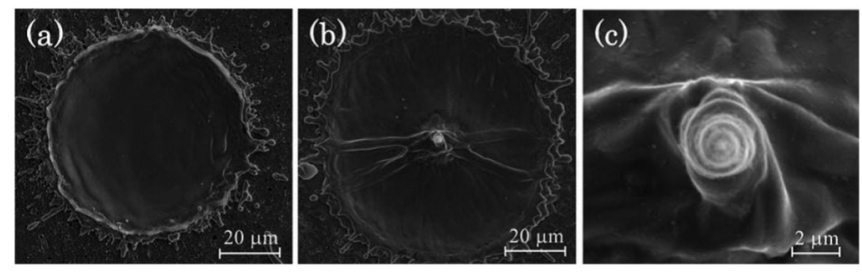

FIG. 1. SEM images of surfaces irradiated by (a) circularly polarized and (b) linearly polarized optical vortex beams. (c) Magnified image of nanoneedle fabricated at center of ablated zone. The needle has a spiral cone.

and the quarter-wave plate. Using this system, the total angular momentum, $J \hbar$, of the light was controlled between $-3 \hbar$ and $3 \hbar$. The numerical aperture of the objective lens in this experiment was tuned in the range of 0.04-0.15 to maintain a constant beam waist of $\omega_{0} \approx$ $33 \mu \mathrm{m}$. The pulse energy was varied in the range of $0.2-0.8 \mathrm{~mJ}$. As in our previous studies $[17,18]$, four vortex pulses were overlaid so as to maximize the nanoneedle height. All experiments were performed at atmospheric pressure and room temperature. The ablated surface of the Ta plate was observed by a scanning electron microscope (SEM; JEOL, JSM-6010LA) with a spatial resolution of $8 \mathrm{~nm}$ at $3 \mathrm{kV}$.

When the target was irradiated with a circularly polarized Gaussian beam with $L=0$ and $S= \pm 1$, no nanostructures, such as needles, were observed on the ablated surface [Fig. 1(a)]. In contrast, a linearly polarized optical vortex with $L \neq 0$ and $S=0$ produced a chiral nanoneedle with a spiral conical surface at the center of the ablation zone [Figs. 1(b) and 1(c)]. As we found in previous studies $[17,18]$, this phenomenon indicates that the optical gradient force collects the melted metal, and the optical scattering force directs it efficiently toward the on-axis core of the optical vortex, thereby confining the melted metal in the core to form the chiral nanoneedle. In Gaussian beams that do not have an on-axis core, the collection of the melted metal does not occur efficiently.

Figures 2(a) and 2(b) show SEM images of chiral nanoneedles fabricated by a linearly polarized first-order optical vortex $(L=1 ; S=0)$ and a second-order optical vortex $(L=2 ; S=0)$, respectively. The nanoneedles are typically $\sim 10 \mu \mathrm{m}$ high relative to the target surface. The tip curvature was typically measured to be $\sim 50 \mathrm{~nm}$, which is approximately $1 / 25$ th of the laser wavelength ( $=1064 \mathrm{~nm}$ ). As the magnitude of $L$ increases, the spiral frequency of the nanoneedle (defined as the number of turns divided by the distance, $h$, between the tip and the bottom of the spiral structure in the chiral nanoneedle), as shown in Fig. 2(f), increased.

Figure 3(a) shows the spiral frequency of the chiral nanoneedle as a function of the magnitude of $L$ of the pump laser. A positive (negative) spiral frequency indicates that the needle is twisted azimuthally in the clockwise (counterclockwise) direction. The spiral
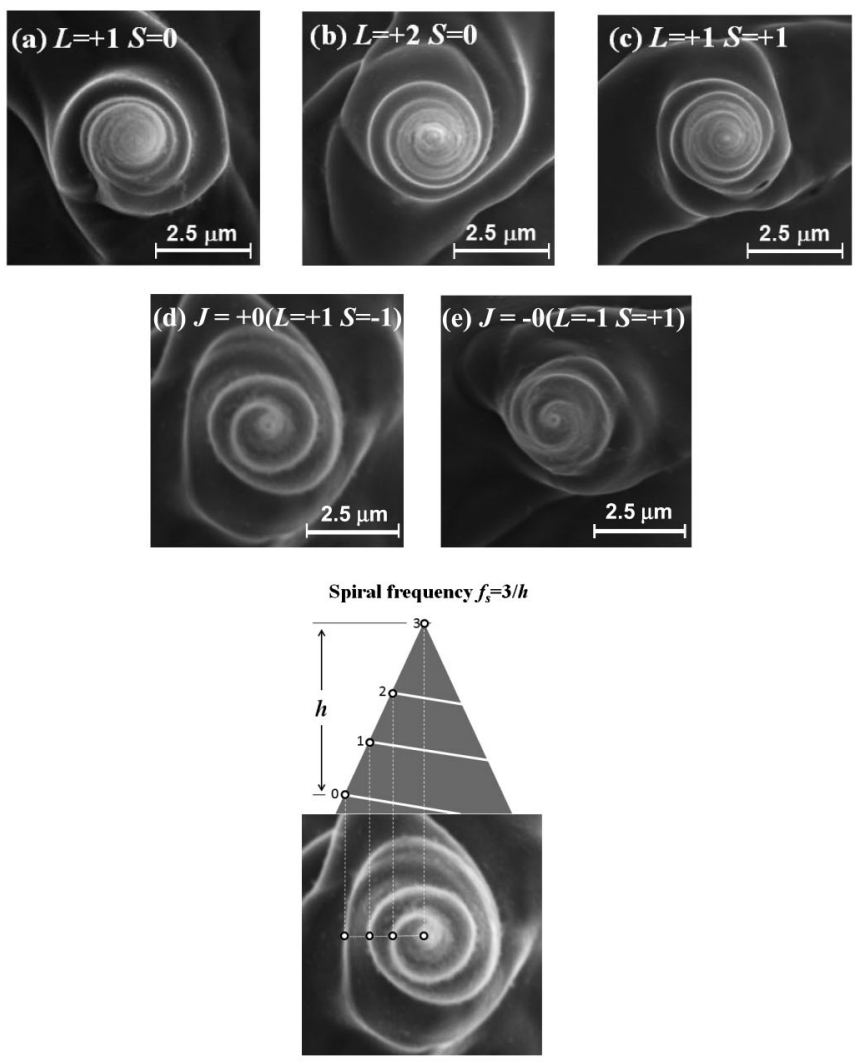

(f)

FIG. 2. Chiral nanoneedles fabricated by (a) linearly polarized first-order and (b) second-order optical vortices. (c) Chiral nanoneedle fabricated by circularly polarized first-order optical vortex with $J=2$. Chiral nanoneedles fabricated by circularly polarized optical vortices with a total angular momentum $J=0: \quad($ d) $\quad L=1, \quad S=-1 \quad$ and $($ e) $\quad L=-1, \quad S=1$. (f) Definition of the spiral frequency of the nanoneedles. The spiral frequency is defined as the number of spiral turns divided by $h$ (distance between the tip and the bottom of the spiral structure in the chiral nanoneedle).

direction (chirality) of the nanoneedles is determined by the sign of $L$; it is not affected by $S$ at all. These results demonstrate that the chirality of the nanoneedle is determined only by the sign of $L . S$ can increase (decrease) the spiral frequency of a nanoneedle when the sign of $L$ is the same as (opposite to) that of $S$. Figure 3(b) shows the spiral frequency of the nanoneedle as a function of $J$ of the pump laser. The maximum spiral frequency of $\sim 0.52 \mu \mathrm{m}^{-1}$ at a pulse energy of $0.8 \mathrm{~mJ}$ was obtained at $J=3$. And even optical vortices with $J=0(L= \pm 1 ; S=\mp 1)$ created chiral nanoneedles [Figs. 2(d) and 2(e)]. At pulse energies higher than the order of millijoules, the spiral structure of the fabricated nanoneedle disappeared, as reported concerning our previous study [18].

For an optical vortex, the spiral frequency of the chiral nanoneedle was determined by the magnitude of $J$, rather than the magnitude of $L$. For example, chiral nanoneedles fabricated by linearly polarized second-order $(L=2 ; S=0)$ and circularly polarized first-order $(L=1 ; S=1)$ optical 


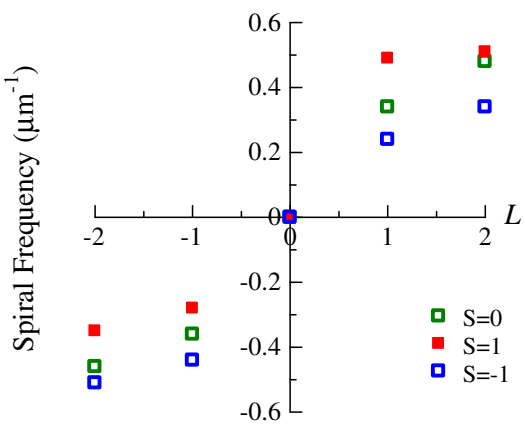

(a)

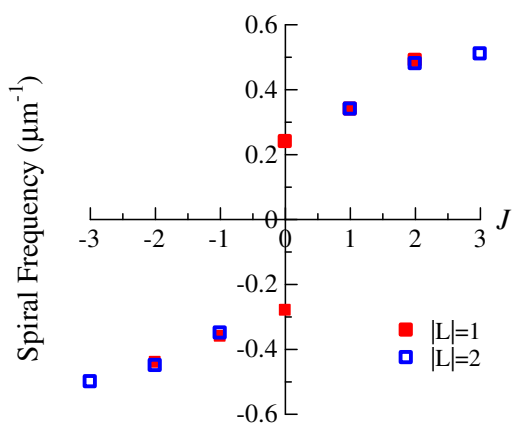

(b)

FIG. 3 (color online). (a) Experimentally obtained plots of spiral frequency as a function of orbital angular momentum $L$ for various spin angular momenta $S(S=1$, black solid boxes; $S=0$, gray open boxes; $S=-1$, black open boxes). (b) Experimentally obtained plots of spiral frequency as a function of orbital angular momentum $J$ for various orbital angular momenta $L$.

vortices have the same chirality and spiral frequency [see Figs. 2(b) and 2(c)]. For all of the pulse energies used in this study, the nanoneedle produced by a linearly polarized second-order optical vortex had the same spiral frequency as that produced by a circularly polarized first-order optical vortex (Fig. 4).

To understand these phenomena (two optical vortices with $J=2$ creating the same chiral nanostructure, polarization helicity reinforcing chiral nanostructure, and even optical vortices with $J=0$ creating nanoneedles), the total angular momentum density of the optical vortices can be examined. The spatial distribution of the total angular momentum density, $j_{L, S}$, of the $L$ th-order optical vortex with $S$ is given by

$$
j_{L, S}(r)=\varepsilon_{0}\left\{\omega L\left|u_{L}\right|^{2}-\frac{1}{2} \omega S r \frac{\partial\left|u_{L}\right|^{2}}{\partial r}\right\},
$$

where $\varepsilon_{0}$ is the dielectric constant of a vacuum, $\left|u_{L}\right|^{2}$ is the optical intensity profile of the $L$ th-order optical vortex, $\omega$ is the frequency of the optical vortex, $\omega_{0}$ is the beam waist of the optical vortex, and $r$ is the radial coordinate of the cylindrical coordinate system [10].

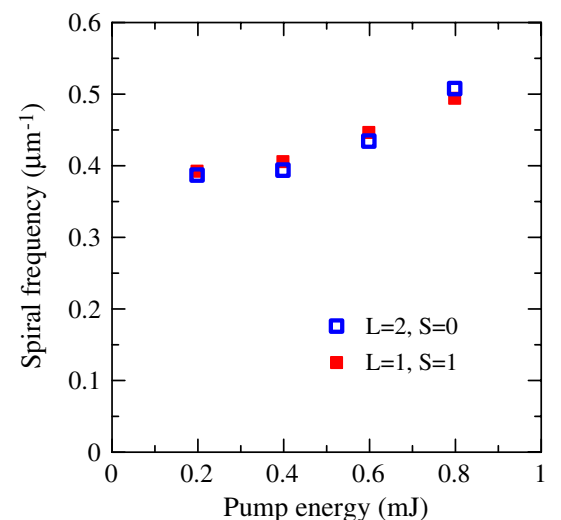

FIG. 4 (color online). Measured spiral frequency of a nanoneedle as a function of the pump energy.

The optical intensity profile, $\left|u_{L}\right|^{2}$, is given by

$$
\left|u_{L}\right|^{2} \propto \frac{1}{|L| !}\left(\frac{\sqrt{2} r}{\omega_{0}}\right)^{2|L|} \exp \left(-2 r^{2} / \omega_{0}^{2}\right),
$$

By substituting Eq. (2) into Eq. (1), the spatial distribution of the total angular momentum density $j_{L, S}(r)$ can be expressed by

$$
\begin{array}{r}
j_{L, S}(r)=l_{z}+s_{z} \propto \frac{\omega}{|L| !}\left[L-|L| S+S\left(\frac{\sqrt{2} r}{\omega_{0}}\right)^{2}\right] \\
\times\left(\frac{\sqrt{2} r}{\omega_{0}}\right)^{2|L|} \exp \left(-\frac{2 r^{2}}{\omega_{0}^{2}}\right), \\
l_{z} \propto \frac{\omega L}{|L| !}\left(\frac{\sqrt{2} r}{\omega_{0}}\right)^{2|L|} \exp \left(-\frac{2 r^{2}}{\omega_{0}^{2}}\right), \\
s_{z} \propto-\frac{\omega S}{|L| !}\left[|L|-\left(\frac{\sqrt{2} r}{\omega_{0}}\right)^{2}\right]\left(\frac{\sqrt{2} r}{\omega_{0}}\right)^{2|L|} \exp \left(-\frac{2 r^{2}}{\omega_{0}^{2}}\right),
\end{array}
$$

A general relationship written as

$$
\begin{gathered}
j_{L, 0}=j_{L-(L /|L|),(L /|L|)} \quad \text { for }|L| \geq 2, \\
j_{L, S}=-j_{-L,-S},
\end{gathered}
$$

can be established. The relationship $j_{2,0}=j_{1,1}$ demonstrates that two optical vortices with $J=2$ are degenerate, and thus produce the same chiral nanostructure. As shown in Fig. 5, the simulated angular momentum densities also indicate that two optical vortices with $J=2$ are degenerate.

The laser energy level used in the present experiments was near the ablation threshold, as described in our previous publication [18]. This low energy permits the metal to melt only in the bright ring of the optical vortex. The melted metal in the bright ring receives effectively the apertured total angular momentum, defined as $\bar{j}_{L, S}(r)=2 \pi \int_{0}^{r} j_{L, S}(r) r d r$ (see Fig. 6). The relationship $j_{1,1}>j_{1,0}>j_{1,-1}$ at large enough $2^{1 / 2} r / \omega_{0}$ is established, 


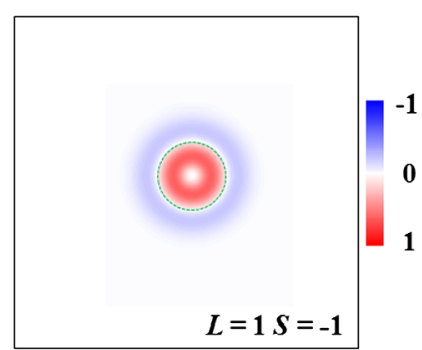

(a)

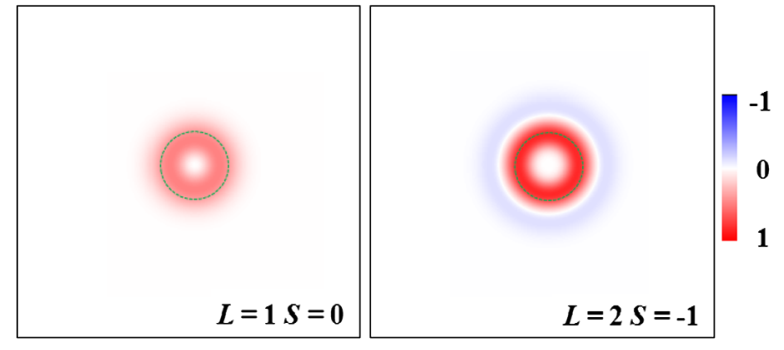

(b)

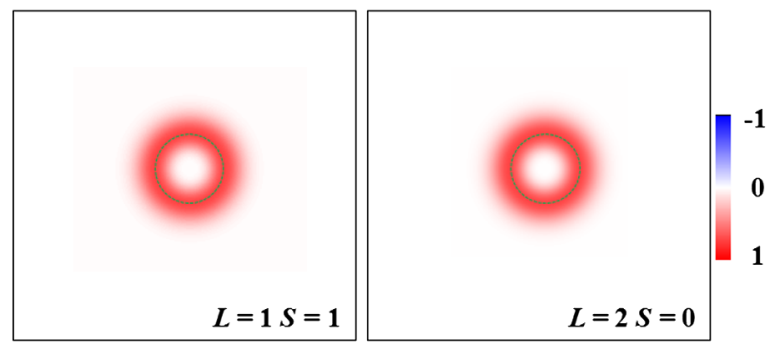

(c)

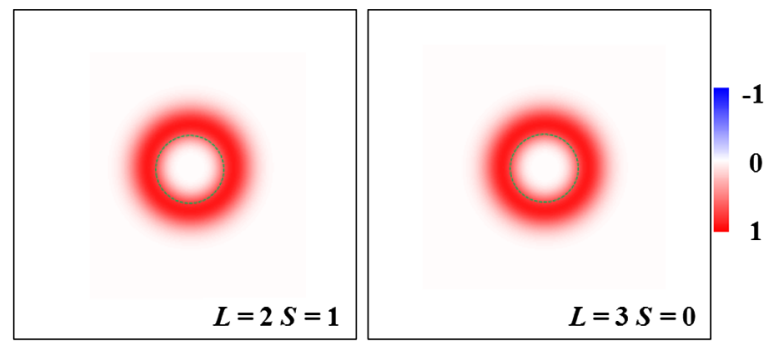

(d)

FIG. 5 (color online). Simulated total angular momentum densities at (a) $J=0$, (b) $J=1$, (c) $J=2$, and (d) $J=3$. The values of total angular momentum densities are normalized by the maximum value of the total angular momentum density at $J=3$. Two optical vortices with $J=2$ (or 3) are degenerate. The optical vortex with $J=0$ exhibits nonzero total angular momentum density near the on-axis core. Thin circles with a radius of $\omega_{0}$ (it means the optical vortex mode field) are also plotted.

and it can well support that $S$ can increase (decrease) the spiral frequency of a nanoneedle when the sign of $L$ is the same as (opposite to) that of $S$.

Furthermore, even though the total angular momentum spatially averaged over the whole beam aperture is zero, a nonzero apertured total angular momentum forces the melted metal to revolve, and its sign is determined by that of the orbital angular momentum, $L \hbar$. Consequently,

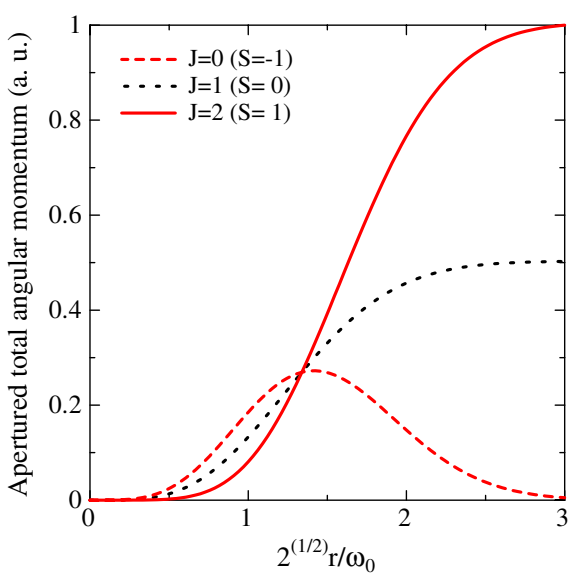

FIG. 6 (color online). Simulated apertured total angular momentum as a function of $r / \omega_{0}$ for $L=1$ and $S=1,0,-1$.

even optical vortices with $J=0$ create chiral nanoneedles. In fact, the relationship $j_{1,-1}=-j_{-1,1}$ from Eq. (7) further indicates that optical vortices with $J=0(L= \pm 1, S=\mp 1)$ can create nanoneedles whose chirality is determined by the sign of $L$ [Figs. 2(d) and 2(e)].

These experimental results suggest the following mechanism. The melted metal upon irradiation of the optical vortex pulse is collected in the ring of the optical vortex by the optical gradient force. The orbital angular momentum of the optical vortex is then transferred to the melted metal, producing orbital motion of the melted metal about the axial core of the optical vortex. The melted metal is also forwardly directed by the optical scattering force, and, subsequently, it is confined in the core (where it does not experience efficiently a forward scattering force and orbital angular momentum because the vortex pulse is almost gone) to produce a chiral needle.

Thus, the chirality of the nanoneedle is determined only by the sign of the orbital angular momentum, $L \hbar$. The polarization helicity (intrinsic helicity) of the optical vortex, which is expected to be $\pm h$ for any aperture, only accelerates (decelerates) the orbital motion of the melted metal, thereby increasing (decreasing) the spiral frequency of the nanoneedle. The resulting spiral frequency of the nanoneedle is also determined by the magnitude of the total angular momentum, $J \hbar$, which is defined as the sum of the orbital and spin angular momenta. In other words, the structure (chirality and spiral frequency) of the fabricated nanoneedles can be used to visualize the helicities of the orbital and total angular momenta of light.

In conclusion, we have investigated the fabrication of chiral nanoneedles by using optical vortices with various angular momenta $(L \hbar, S \hbar$, and $J \hbar)$. The orbital angular momentum of the optical vortex, transferred to the material during irradiation of the optical vortex pulse, mainly determines the chirality of the nanostructures. The polarization helicity of the optical vortex also accelerates (decelerates) the orbital motion of the melted metal to increase 
(decrease) the spiral frequency of the chiral nanoneedle. Because optical vortices with the same value of $J(=2)$ are degenerate, they create the nanoneedle with the same chirality. Chiral nanoneedle formation involving transfer of the helicity of the incident light to a material can be used to visualize the wave front helicity of the orbital angular momentum of the light. The polarization helicity of the optical vortex can reinforce the helical structure of the created chiral nanoneedles. Control of the chirality of nanostructures produced by light has the potential to produce new structures such as planar chiral metamaterials, plasmonic nanostructures, and selective identification of chiral chemical composites.

The authors acknowledge support from a Grant-in-Aid for Scientific Research (No. 21360026) from the Japan Society for the Promotion of Science. We would also like to thank Professor E. Brasselet from Université Bordeaux 1, France, for his productive suggestions.

[1] L. Allen, M. W. Beijersbergen, R. J. C. Spreeuw, and J. P. Woerdman, Phys. Rev. A 45, 8185 (1992).

[2] M. Padgett, J. Courtial, and L. Allen, Phys. Today 57, No. 5, 35 (2004).

[3] G. Indebetouw, J. Mod. Opt. 40, 73 (1993).

[4] S. Franke-Arnold, L. Allen, and M. J. Padgett, Laser Photon. Rev. 2, 299 (2008).

[5] K. T. Gahagan and G. A. Swartzlander, Jr., Opt. Lett. 21, 827 (1996).

[6] N. B. Simpson, K. Dholakia, L. Allen, and M. J. Padgett, Opt. Lett. 22, 52 (1997).

[7] L. Paterson, M. P. MacDonald, J. Arlt, W. Sibbett, P. E. Bryant, and K. Dholakia, Science 292, 912 (2001).

[8] S. Bretschneider, C. Eggeling, and S. W. Hell, Phys. Rev. Lett. 98, 218103 (2007).
[9] T. Watanabe, Y. Iketaki, T. Omatsu, K. Yamamoto, M. Sakai, and M. Fujii, Opt. Express 11, 3271 (2003).

[10] A. T. O’Neil, I. MacVicar, L. Allen, and M. J. Padgett, Phys. Rev. Lett. 88, 053601 (2002).

[11] Q. Zhan, Opt. Lett. 31, 867 (2006).

[12] Y. Zhao, J. S. Edgar, G. D. M. Jeffries, D. McGloin, and D. T. Chiu, Phys. Rev. Lett. 99, 073901 (2007).

[13] G. M. Lerman, A. Yanai, and U. Levy, Nano Lett. 9, 2139 (2009).

[14] B. Desiatov, I. Goykhman, and U. Levy, Nano Lett. 9, 3381 (2009).

[15] A. Yanai and U. Levy, Opt. Express 17, 924 (2009).

[16] J. Hamazaki, R. Morita, K. Chujo, Y. Kobayashi, S. Tanda, and T. Omatsu, Opt. Express 18, 2144 (2010).

[17] T. Omatsu, K. Chujo, K. Miyamoto, M. Okida, K. Nakamura, N. Aoki, and R. Morita, Opt. Express 18, 17967 (2010).

[18] K. Toyoda, K. Miyamoto, N. Aoki, R. Morita, and T. Omatsu, Nano Lett. 12, 3645 (2012).

[19] N. M. Litchinitser, Science 337, 1054 (2012).

[20] K. Konishi, B. Bai, X. Meng, P. Karvinen, J. Turunen, Y. P. Svirko, and M. Kuwata-Gonokami, Opt. Express 16, 7189 (2008).

[21] S. Zhang, Y. S. Park, J. Li, X. Lu, W. Zhang, and X. Zhang, Phys. Rev. Lett. 102, 023901 (2009).

[22] M. I. Stockman, Phys. Rev. Lett. 93, 137404 (2004).

[23] P. Nagpal, N.C. Lindquist, S. H. Oh, and D. J. Norris, Science 325, 594 (2009).

[24] R. Garcia, R. V. Martinez, and J. Martinez, Chem. Soc. Rev. 35, 29 (2006).

[25] R. D. Piner, J. Zhu, F. Xu, S. Hong, and C. A. Mirkin, Science 283, 661 (1999).

[26] G. Binnig, H. Rohrer, Ch. Gerber, and and E. Weibel, Phys. Rev. Lett. 49, 57 (1982).

[27] H. Dai, J. H. Hafnar, A. G. Rinzler, D. T. Colbert, and R. E. Smalley, Nature (London) 384, 147 (1996).

[28] L. Torrisi, F. Caridi, A. Picciotto, D. Margarone, and A. Borrielli, J. Appl. Phys. 100, 093306 (2006). 